\title{
Adaptações na gestão de unidades de alimentação e nutrição durante a pandemia da
}

\section{Covid-19}

\author{
Adaptations in the management of food and nutrition units during the Covid-19 pandemic \\ Adaptaciones en la gestión de las unidades de alimentos y nutrición durante la pandemia Covid-19
}

Recebido: 17/09/2021 | Revisado: 29/09/2021 | Aceito: 01/10/2021 | Publicado: 03/10/2021

\author{
Juliana da Silveira \\ ORCID: https://orcid.org/0000-0001-9659-4680 \\ Pontifícia Universidade Católica do Paraná, Brasil \\ E-mail: silveira7juliana@gmail.com \\ Cilene da Silva Gomes Ribeiro \\ ORCID: https://orcid.org/0000-0002-1899-4408 \\ Pontifícia Universidade Católica do Paraná, Brasil \\ E-mail: cilenex@hotmail.com \\ Letícia Caroline dos Santos Gimenes \\ ORCID: https://orcid.org/0000-0002-3417-9593 \\ Pontifícia Universidade Católica do Paraná, Brasi \\ E-mail: leticiacaroline8@hotmail.com
}

\begin{abstract}
Resumo
O objetivo do estudo foi analisar as adaptações na gestão de colaboradores, de matérias-primas e de boas práticas de fabricação durante a pandemia da Covid-19 em unidades de alimentação e nutrição (UAN) de dois segmentos: o comercial e o industrial/corporativo. A pesquisa se deu com gestores de UAN de todo o Brasil, por meio de questionário online. Foi identificado que os dois segmentos foram afetados pela pandemia da Covid-19, sendo os comerciais os que mais fizeram jus a medida trabalhista provisória $n^{\circ} 936 / 2020$. Em adição, o setor comercial foi o mais afetado pelo desperdício de matéria prima e a necessidade de mudança de fornecedores durante a pandemia. Mas, foram as UAN industriais/corporativas que tiveram mais facilidades nas adequações higiênicas sanitárias. Muitos são os fatores que geraram crescentes falências neste segmento, mas a falta de conhecimentos e práticas gerenciais competentes anteriores, impactaram sobremaneira as consequências durante a pandemia.
\end{abstract}

Palavras-chave: Gestão; Restaurantes; Covid-19; Boas práticas de fabricação.

\begin{abstract}
The objective of the study was to analyze the adaptations in the management of raw materials and good manufacturing practices during the Covid-19 pandemic in food and nutrition units (FNU) in two segments: commercial and industrial/corporate. The research was carried out with FNU managers from all over Brazil, through an online survey. It was identified that both segments were affected by the Covid-19 pandemic, with the commercial ones being the most entitled to provisional labor measure No. 936/2020. In addition, the commercial sector was the most affected by the waste of raw materials and the need to change suppliers during the pandemic. However, it was the industrial/corporate FNU that had more facilities in sanitary hygiene adjustments. There are many factors that have generated increasing failures in this segment, but the lack of previous knowledge and competent managerial practices greatly impacted the consequences during the pandemic.
\end{abstract}

Keywords: Management; Restaurants; Covid-19; Good manufacturing practices.

\section{Resumen}

El objetivo del estudio fue analizar las adaptaciones en la gestión de los empleados, las matérias primas y las buenas prácticas de fabricación durante la pandemia da Covid-1 en unidades de alimentación y nutrición (UAN) de dos segmentos: el comercial y el industrial/corporativo. La encusta ocurrió con los gerentes de UAN de todo Brasil, a través de un cuestionario en línea. Fue identificado que los dos segmentos se vieron afectados por la pandemia de Covid-19, siendo los comerciantes que más han utilizado la medición del trabajo provisional nº 936/2020. Además, el sector comercial se vio muy afectado por el desperdicio de materia prima y la necesidad de cambiar de proveedores durante la pandemia. Pero, fueron las UAN industrial / corporativa que tenían más instalaciones en condiciones higiénico-sanitarias Muchos son los factores que han generado quiebras crecientes en este segmento, pero la falta de conocimientos y prácticas de gestión competentes anteriores han impactado en gran medida las consecuencias durante la pandemia.

Palabras clave: Administración; Restaurante; Covid-19; Buenas prácticas de fabricación. 


\section{Introdução}

O SARS-Cov-2 coronavírus zoonótico da síndrome respiratória aguda grave 2, que desencadeia a Covid-19 (do inglês Coronavírus Disease 2019) é altamente contagiosa entre humanos (Kumar, Taki, Gahlot, Sharma \& Dhangar, 2020) e pode persistir por horas em diferentes superfícies inanimadas, como por exemplo metal, vidro e plástico (Kampf, Pfaender \& Steinmann, 2020). Estimativas da OMS indicam que mais de 4,5 milhões de pessoas já vieram a óbito até setembro de 2021, em virtude da Covid-19 (https://www.who.int/emergencies/diseases/novel-coronavirus-2019 recuperado em 01, setembro, 2021), sendo mais de 580 mil pessoas somente do Brasil (https://covid.saude.gov.br/ recuperado em 01, setembro, 2021).

Para limitar a transmissão da doença, antes da vacinação da maioria da população, e para evitar o colapso do sistema de saúde, tem sido necessárias medidas de contenção como, por exemplo, o isolamento de doentes (Lei 13.979, 2020), e/ou de pessoas em grupo de risco e/ou a redução da circulação de pessoas em espaços coletivos públicos e privados. Desse modo, entre essas medidas, a última tem sido a que mais interfere na economia (Ozili, \& Arun, 2020; Song, Yeon, \& Lee, 2021).

Em diferentes regiões do mundo, autoridades governamentais decretaram o fechamento de diversos setores da economia, em diferentes momentos da pandemia, exceto aqueles considerados essenciais, como medida protetiva no intuito de promover o isolamento social. Logo, empresas de divergentes áreas implementaram o trabalho remoto (home office) para manterem suas atividades (VoPham et al., 2020; Hakim, Zanetta \& da Cunha, 2021). Contudo, muitos negócios não conseguiram adotar esse modelo de trabalho para todos os cargos da empresa, e precisaram parar, reduzir algumas atividades e/ou utilizar auxílios provenientes do Estado para manter o pagamento e o vínculo empregatício dos funcionários (Medida provisória 927, 2020; Medida provisória 936, 2020).

Alguns dos empreendimentos que foram amplamente afetados foram aqueles que produzem e distribuem alimentos, também denominados Unidades de Alimentação e Nutrição (UAN), como exemplo, restaurantes, bares, panificadoras, lanchonetes, rotisserias e congêneres (Resolução da Diretoria Colegiada 216, 2004), uma vez que conforme as medidas de combate ao Sars-CoV-2, foram proibidos de servir alimentos para consumo no local (Song et al., 2021).

Desse modo, apesar de alguns estabelecimentos terem adotado o sistema delivery, auxiliando no escoamento de suas produções, no Brasil, há estimativas que a receita de 53,2\% de empreendimentos que produzem e fornecem refeição fora do lar, do início da pandemia até abril de 2020, tenha diminuído em torno de 50 a $90 \%$ (Associação Nacional de Restaurantes, 2020). Nos EUA, a indústria de restaurantes perdeu mais de 120 bilhões de dólares em vendas, e 2 a cada 3 funcionários foram demitidos ou dispensados em maio de 2020 (National Restaurant Association, 2020). Na Índia, as perdas no setor de restaurantes chegaram a 1 trilhão de rúpias em meio a pandemia (Vig \& Agarwal, 2021).

Com o afrouxamento das restrições, que foi realizada diversas vezes conforme sistema de bandeiras, alguns empreendimentos puderem retornar com suas atividades desde que seguissem determinadas orientações higiênico sanitárias para poder atender os clientes de forma presencial. Entretanto, destaca-se que existem diferentes segmentos nos quais os empreendimentos que produzem e distribuem refeições podem atuar, e compreender como foi realizada a gestão de cada um deles durante a pandemia é necessário para identificar problemas e antecipar medidas para situações semelhantes que possam vir a surgir.

Diante disso, o objetivo do estudo foi analisar as adaptações, na gestão de colaboradores, de matérias-primas e de boas práticas de fabricação, durante a pandemia da Covid-19, em Unidades de Alimentação e Nutrição de dois segmentos: o comercial, com atendimento ao público em geral, e do segmento industrial, que são aqueles vinculados a corporações e possuem atendimento ao público direcionado. 


\section{Metodologia}

O presente estudo, aprovado pelo Comitê de Ética em pesquisa da Pontifícia Universidade Católica do Paraná (número de parecer 4.137.982), foi realizado com gestores de Unidades de Alimentação e Nutrição de diferentes regiões do Brasil, durante $1^{\circ}$ de agosto a $1^{\circ}$ setembro de 2020, sendo que nesse período o país apresentava 93.563 óbitos e 2.707.877 casos de Covid-19 na primeira data, e 122.596 óbitos e 3.950.931 casos na última data (https://covid.saude.gov.br/ recuperado em 06, julho, 2021).

Uma vez que a pesquisa foi feita de modo online, o convite para o estudo e o questionário foram vinculados e divulgados em plataformas de redes sociais, entre as quais o Facebook ${ }^{\circledR}$, Whatsapp ${ }^{\circledR}$ e Linkedin ${ }^{\circledR}$. Esse questionário compreendeu 67 perguntas divididas em quatro etapas, referentes à profissão e local de trabalho do gestor, mudanças relativas ao quadro de colaboradores das UAN durante a pandemia, adaptações referentes aos alimentos fornecidos e modificações em protocolos de Boas Práticas de Fabricação (BPF). Destaca-se que apenas os participantes que responderam todas as questões foram incluídos no estudo.

$\mathrm{Na}$ análise estatística, para verificar a normalidade e a homogeneidade dos resultados empregou-se o teste de Shapiro-wilk e teste F, respectivamente. Em seguida, os dados quantitativos foram submetidos ao teste Mann-Whitney e os qualitativos ao teste do qui-quadrado. As análises foram feitas com o auxílio do programa Statistical Package for the Social Sciences (SPSS).

\section{Resultados e Discussão}

Das 105 UAN analisadas, 92,4\% atuavam na região Sul do Brasil e 72,4\% apresentavam gestores nutricionistas, conforme demonstra a Tabela 1. Quanto à classificação das UAN segundo o número de refeições servidas por dia (Sant'Ana, 2012), observou-se que 57,1\% descreveram servir à época até 500 refeições por dia (porte pequeno), 35,2\% até 2000 mil (porte médio) e 7,6\% entre 2000 a 10000 (porte grande). Em adição, pelo segmento de atuação, 69,5\% das UAN relataram atuar no setor corporativo/ industrial e 30,5\% no comercial. 
Tabela 1. Perfil dos gestores e das unidades de alimentação e nutrição avaliadas, durante o período de agosto a setembro de 2021.

\begin{tabular}{|c|c|}
\hline Variável & Valores em \% (n) \\
\hline \multicolumn{2}{|l|}{ Profissão do gestor das UAN } \\
\hline Nutricionista & $72,4(76)$ \\
\hline Empresário & $8,6(9)$ \\
\hline Administrador & $1,9(2)$ \\
\hline Chefe de cozinha & $3,8(4)$ \\
\hline Gastrônomo & $4,8(5)$ \\
\hline Técnico de nutrição & $1,9(2)$ \\
\hline Outros & $6,7(7)$ \\
\hline Total & $100(105)$ \\
\hline \multicolumn{2}{|l|}{ Região geográfica onde se encontrava a UAN } \\
\hline Sul & $92,4(97)$ \\
\hline Sudeste & $4,8(5)$ \\
\hline Nordeste & $1,9(2)$ \\
\hline Norte & $0,9(1)$ \\
\hline Centro-Oeste & $0(0)$ \\
\hline Total & $100(105)$ \\
\hline \multicolumn{2}{|l|}{ Porte das UAN } \\
\hline Pequeno (até 500 refeições/ dia) & $57,1(60)$ \\
\hline Médio (501 a 2000 refeições/ dia) & $35,2(37)$ \\
\hline Grande (2001 a 10000 refeições/ dia) & $7,6(8)$ \\
\hline Extra (acima de 10000 refeições/ dia) & $0(0)$ \\
\hline Total & $100(105)$ \\
\hline \multicolumn{2}{|l|}{ Classificação dos segmentos das UAN } \\
\hline Corporativo/industrial & $69,5(73)$ \\
\hline Comercial & $30,5(32)$ \\
\hline Total & $100(105)$ \\
\hline \multicolumn{2}{|l|}{ Número de funcionários totais por segmento } \\
\hline Corporativo/industrial & $62,1(1761)$ \\
\hline Comercial & $37,9(1075)$ \\
\hline Total & $100(2836)$ \\
\hline
\end{tabular}

Fonte: Autores (2021).

Um maior número de estabelecimentos provenientes do estado do Paraná, Santa Catarina e Rio Grande do Sul, pode ser explicado pelo método de divulgação da pesquisa adotado para esse estudo. O questionário online foi divulgado por meio das redes sociais das pesquisadoras, sendo que estas apresentavam maior número de contatos provenientes da região sul.

A maior presença de gerentes nutricionistas nos restaurantes, se deve ao fato de que estes são formados para supervisionar a produção, treinar funcionários, gerenciar custos, gastos e lucros, promover a educação nutricional, além de serem responsáveis técnicos desses estabelecimentos (Lei 8.234, 1991; Cunha, Rosso \& Stedefeldt, 2018). Contudo, uma vez que os processos dos restaurantes são comuns na área da saúde, ciência dos alimentos e administração, diferentes profissões são capacitadas para ocupar o cargo de gerente, como os administradores, gastrônomos, chefes de cozinhas e engenheiros de alimentos.

Logo, o sucesso das UAN quanto ao faturamento e a qualidade dos alimentos depende principalmente das decisões tomadas pelos gestores. Essas decisões normalmente são conhecidas ou previsíveis, como por exemplo, a falta de funcionários, atraso de matéria prima, acidentes de trabalhos, mudanças de cardápios, entre outras. Porém, durante a pandemia da Covid-19 circunstâncias jamais vivenciadas influenciaram nas estratégias utilizadas pelos gestores dentro das UAN. Uma das primeiras 
situações foi a possibilidade de contaminação de clientes e funcionários, a necessidade de isolamento social e o fechamento e/ou a paralisação de atividades não essenciais (Lei 13.979, 2020; Ozili, \& Arun, 2020; Song, Yeon, \& Lee, 2021).

Conforme a Tabela 2, verifica-se que 36,2\% das UAN paralisaram suas atividades, sendo um maior número de estabelecimentos comerciais $(65,6 \%)$ em comparação ao segmento corporativo/industrial $(23,3 \%)$ ( $<<0,05)$. Isso pode ter ocorrido em virtude de que as UAN comerciais encerraram, paralisaram, reduziram e ou alteraram suas atividades mais de uma vez durante a pandemia, conforme o risco de colapso do sistema de saúde e os sistemas de sinalização.

Tabela 2. Adequações de funcionamento, atendimento e de gastos, relatadas pelas UAN, do início da pandemia até 01 de setembro de 2021.

\begin{tabular}{cccc}
\hline Segmento & $\begin{array}{c}\text { Paralisação de } \\
\text { atividades } \\
\boldsymbol{\%}(\mathbf{n})\end{array}$ & $\begin{array}{c}\text { Atendimento no } \\
\text { sistema delivery } \\
\boldsymbol{\%}(\mathbf{n})\end{array}$ & $\begin{array}{c}\text { Restrição de gastos } \\
\text { \%(n) }\end{array}$ \\
\hline Corporativo/industrial (73) & $23,3(17)^{\mathrm{a}^{*}}$ & $6,9(5)^{\mathrm{a}}$ & $72,6(53)^{\mathrm{a}}$ \\
Comercial (32) & $65,6(21)^{\mathrm{b}}$ & $59,4(19)^{\mathrm{b}}$ & $71,9(23)^{\mathrm{a}}$ \\
Total (105) & $36,2(38)$ & $22,9(24)$ & $72,4(76)$ \\
\hline
\end{tabular}

*Letras iguais, na mesma coluna não apresentaram diferença significativa conforme determinado pelo teste do qui-quadrado ( $p>0,05$ ). Fonte: Autores (2021).

Por outro lado, os estabelecimentos do segmento corporativo/industrial funcionaram de acordo com suas contratantes, que na maioria dos casos são indústrias classificadas como de atividades essenciais, e por isso não tiveram seus ofícios paralisados por longos períodos. Entretanto, inevitavelmente também ocorreram reduções na venda de refeições e de atendimento nos empreendimentos corporativos/industriais, pois muitos funcionários das empresas contratantes passaram a exercer suas atividades em home office, entraram em férias e ou tiveram carga horário reduzida (Da Silva, Carvalho \& Mendes, 2021; Oliveira, Abranches \& Lana 2020).

Com a diminuição de clientes presenciais, e para ajudar no escoamento da produção, alguns estabelecimentos passaram a funcionar com sistema delivery (Oliveira et al., 2020). Entretanto, observa-se que um maior número de estabelecimentos comerciais, em comparação ao corporativo/industrial relataram à época entregar refeições em domicílio. Essa diferença se deve em razão de que os segmentos corporativos/indústrias são contratados para servir refeição para os colaboradores da empresa contratante somente durante o período de trabalho, além disso não possuem flexibilidade para produzir e distribuir refeições a longas distâncias para os funcionários que aderiram ao trabalho remoto.

Apesar de um menor número de estabelecimentos corporativos/indústrias relatarem terem paralisado suas atividades em relação ao segmento comercial, e que esse último funcionou no sistema delivery em maior número do que o primeiro, $72,4 \%$ dos gestores relataram a época estarem diminuindo gastos, sendo 72,6\% nas UAN corporativo/industrial e 71,9\% nos estabelecimentos comerciais. Logo, verifica-se que a pandemia da Covid-19 atingiu financeiramente ambos os segmentos $(\mathrm{p}>0,05)$.

Desde modo, com intuito de reduzir ainda mais os impactos dos gastos de produção, os gestores de ambos os segmentos puderam requerer medidas trabalhistas provisórias, criadas pelo governo federal com o intuito de preservar o emprego e a renda dos funcionários, entre as quais estão a antecipação de férias, suspensão do contrato de trabalho e redução proporcional de jornada de trabalho e de salário (Medida Provisória 927, 2020; Medida Provisória 936, 2020).

Observa-se na Tabela 3, que os gestores optaram principalmente por suspender o contrato e reduzir proporcionalmente a jornada de trabalho e o salário de seus funcionários em comparação a demissão, antecipação de férias e compensação de horas extras. Além disso, um maior número de estabelecimentos do setor corporativo/industrial $(80,8 \%)$ antecipou as férias de seus funcionários em comparação ao comercial $(53,1 \%)(\mathrm{p}<0,05)$. Por outro lado, um maior número de 
estabelecimentos comerciais $(68,8 \%)$ reduziu proporcionalmente a jornada de trabalho e o salário de seus funcionários em comparação ao setor corporativo/industrial $(37 \%)(\mathrm{p}<0,05)$.

Tabela 3. Medidas trabalhistas adotadas pelas UAN, em referência ao segmento e ao número de estabelecimentos, do início da pandemia até 01 de setembro de 2021.

\begin{tabular}{cccccc}
\hline Segmento & $\begin{array}{c}\text { Demissão } \\
\%(\mathbf{n})\end{array}$ & $\begin{array}{c}\text { Antecipação } \\
\text { de férias } \\
\%(\mathbf{n})\end{array}$ & $\begin{array}{c}\text { Compensação } \\
\text { de horas extras } \\
\%(\mathbf{n})\end{array}$ & $\begin{array}{c}\text { Suspenção } \\
\text { temporária do } \\
\text { contrato de } \\
\text { trabalho** } \\
\%(\mathbf{n})\end{array}$ & $\begin{array}{c}\text { Redução } \\
\text { proporcional } \\
\text { da jornada de } \\
\text { trabalho e de } \\
\text { salário** } \\
\%(\mathbf{n})\end{array}$ \\
\hline Corporativo/Industrial (73) & $58,9(43)^{\mathrm{a}^{*}}$ & $80,8(59)^{\mathrm{a}}$ & $23,3(17)^{\mathrm{a}}$ & $58,9(43)^{\mathrm{a}}$ & $37(27)^{\mathrm{b}}$ \\
Comercial (32) & $53,1(17)^{\mathrm{a}}$ & $53,1(17)^{\mathrm{b}}$ & $28,1(9)^{\mathrm{a}}$ & $68,8(22)^{\mathrm{a}}$ & $68,8(22)^{\mathrm{a}}$ \\
Total (105) & $57,1(60)$ & $72,4(76)$ & $24,8(26)$ & $61,9(65)$ & $46,7(49)$ \\
\hline
\end{tabular}

*Letras iguais, na mesma coluna não apresentaram diferença significativa conforme determinado pelo teste do qui-quadrado ( $\mathrm{p}>0,05)$.

***Medida provisória $\mathrm{n}^{\circ} 936$, de $1^{\circ}$ de abril de 2020.

Fonte: Autores (2021).

Ao comparar os resultados das medidas trabalhistas adotadas pelos gestores, em referência ao número de funcionários (Tabela 4), verifica-se que os estabelecimentos, no geral, apresentaram maior porcentagem de funcionários que tiveram férias antecipadas $(40,7 \%)$ em comparação às outras opções. Além disso, ao comparar por segmentos, os estabelecimentos comerciais tiveram significativamente $(\mathrm{p}<0,05)$ mais colaboradores com o contrato de trabalho suspenso $(49,8 \%)$ e com redução proporcionalmente da jornada de trabalho e salário $(61,1 \%)$ em comparação ao segmento corporativo/industrial, visto que esse último apresentou $15,3 \%$ e $18,5 \%$ respectivamente.

Tabela 4. Medidas trabalhistas adotadas pelas UAN, em referência ao segmento e ao número de funcionários, do início da pandemia até 01 de setembro de 2021.

\begin{tabular}{cccccc}
\hline Segmento & $\begin{array}{c}\text { Demissão } \\
\%(\mathbf{n})\end{array}$ & $\begin{array}{c}\text { Antecipação de } \\
\text { férias } \\
\%(\mathbf{n})\end{array}$ & $\begin{array}{c}\text { Compensação } \\
\text { de horas extras } \\
\%(\mathbf{n})\end{array}$ & $\begin{array}{c}\text { Suspensão } \\
\text { temporária } \\
\text { do contrato } \\
\text { de trabalho** } \\
\text { \%(n) }\end{array}$ & $\begin{array}{c}\text { Redução } \\
\text { proporcional } \\
\text { de jornada de } \\
\text { trabalho e de } \\
\text { salário** } \\
\%(\mathbf{n})\end{array}$ \\
\hline Corporativo/Industrial $(1761)$ & $14,2(250)^{\mathrm{a}}$ & $29,8(524)^{\mathrm{a}}$ & $11,5(202)^{\mathrm{a}}$ & $15,3(270)^{\mathrm{b}}$ & $18,5(325)^{\mathrm{b}}$ \\
Comercial $(1075)$ & $29,1(313)^{\mathrm{a}}$ & $58,6(630)^{\mathrm{a}}$ & $37,5(403)^{\mathrm{a}}$ & $49,8(535)^{\mathrm{a}}$ & $61,1(657)^{\mathrm{a}}$ \\
Total $(2836)$ & $19,9(563)$ & $40,7(1154)$ & $21,3(605)$ & $28,4(805)$ & $34,6(982)$ \\
\hline
\end{tabular}

*Letras iguais, na mesma coluna não apresentaram diferença significativa conforme determinado pelo teste do Mann-Whitney ( $p>0,05$ ).

**Medida provisória $\mathrm{n}^{\circ} 936$, de $1^{\circ}$ de abril de 2020

Fonte: Autores (2021).

Com os resultados obtidos, é possível explicar o motivo que levou os gestores do segmento comercial a optaram por suspender temporariamente o contrato de trabalho e reduzir a jornada de trabalho e salário de seus colaboradores, uma vez que apesar de não estarem funcionando para atendimento local, poderiam adotar o sistema delivery de distribuição dos alimentos e refeições produzidas. Já o segmento industrial/corporativo preferiu antecipar as férias de seus colaboradores, principalmente para que se respeitasse o período de quarentena e/ou como alternativa para diminuir o fluxo de funcionários dentro desses estabelecimentos, visto que o número de clientes também estava reduzido.

Destaca-se que a compensação de horas extras foi uma das medidas menos utilizada pelos gestores, visto que apenas $24,8 \%$ das UAN relataram utilizar essa medida, e que $21,3 \%$ dos funcionários tiveram as horas extras compensadas. Isso pode 
ter ocorrido, pois a compensação de horas extras, dificilmente dura o período necessário para completar a quarentena e não diminui de forma significativa os custos de mão de obra.

A quantidade de demissões de funcionários totais observada neste estudo $(19,9 \%)$ foi menor do publicado pela Associação Nacional de Restaurantes em parceria com a empresa Galunion e o Instituto Foodservice Brasil (2020) que foi de $37 \%$ (Dados coletados entre novembro a dezembro de 2020). Tal divergência nos dados pode ser explicada pela situação pandêmica apresentada em cada momento de ambas as pesquisas. Desse modo, com a continuação da pandemia e sem previsão de quantitativos de vacina para toda a população, as demissões nos empreendimentos pesquisados neste estudo, podem ter sido bem superiores. Outrossim, destaca-se que pesquisa feita novamente pela Associação Nacional de Restaurantes em parceria com a empresa Galunion e o Instituto Foodservice Brasil (2021), entre abril a maio de 2021, indica que 21\% dos funcionários de empreendimentos fora do lar foram demitidos, sendo esse valor mais próximo ao reportado no presente estudo.

Por fim, verificou-se que independente do segmento, todas as UAN apresentaram perda ou déficit de mão de obra ativa. Essa diminuição de funcionários nos processos de produção, seja pela redução da carga-horária, por férias ou por doença, acabou por sobrecarregando o trabalho de outros colaboradores, uma vez que para produzir refeições, independentemente da quantidade produzida e do tipo de atividade, são necessárias as mesmas dispensações de força de trabalho e tempo (Colares \& Freitas, 2007).

Desse modo, para a produção ocorrer sem eventuais problemas e de forma harmonizada, o quadro de funcionários e o orçamento devem estar de acordo com o cardápio e o número de refeições e preparações servidas. Logo, ao analisar a Tabela 5 , é possível imaginar que a alteração de cardápio e a redução do número de preparações servidas, observada em 69,5\% e 68,5\%, respectivamente, dos estabelecimentos, ocorreram para facilitar o trabalho dos funcionários e evitar sobras devido a diminuição de clientes. Entretanto, outro motivo que pode ter levado as alterações do cardápio, em ambos os segmentos, foi o aumento dos preços dos alimentos verificados nesse período da pandemia (Bucak \& Yiğit, 2020).

Tabela 5. Modificações do fornecimento de refeições e problemas relacionados a aquisição de matérias primas apresentadas pelas UAN, do início da pandemia até 01 de setembro de 2021.

\begin{tabular}{|c|c|c|c|c|c|c|}
\hline Segmento & $\begin{array}{l}\text { Alteração } \\
\text { de } \\
\text { cardápio } \\
\%(\mathbf{n})\end{array}$ & $\begin{array}{l}\text { Redução do } \\
\text { número de } \\
\text { preparações } \\
\text { servidas } \\
\%(n)\end{array}$ & $\begin{array}{c}\text { Desperdício de } \\
\text { matéria prima } \\
\%(\mathbf{n})\end{array}$ & $\begin{array}{c}\text { Entrega de } \\
\text { matérias } \\
\text { primas } \\
\text { cancelada } \\
\text { pelos } \\
\text { fornecedores } \\
\%(n) \\
\end{array}$ & $\begin{array}{l}\text { Mudança de } \\
\text { fornecedores } \\
\text { durante a } \\
\text { pandemia } \\
\%(n)\end{array}$ & $\begin{array}{l}\text { Aumento dos } \\
\text { gastos com } \\
\text { materiais } \\
\text { descartáveis } \\
\%(n)\end{array}$ \\
\hline Corporativo/Industrial (73) & $71,2(52)^{\mathrm{a}^{*}}$ & $65,8(48)^{\mathrm{a}}$ & $31,5(23)^{b}$ & $19,2(14)^{\mathrm{a}}$ & $16,4 \%(12)^{\mathrm{b}}$ & $95,9(70)^{\mathrm{a}}$ \\
\hline Comercial (32) & $65,6(21)^{\mathrm{a}}$ & $75(24)^{\mathrm{a}}$ & $56,3(18)^{\mathrm{a}}$ & $28,1(9)^{\mathrm{a}}$ & $40,6 \%(13)^{\mathrm{a}}$ & $84,4(27)^{a}$ \\
\hline Total (105) & $69,5(73)$ & $68,5(72)$ & $39,0(41)$ & $21,9(23)$ & $23,8 \%(25)$ & $92,4(97)$ \\
\hline
\end{tabular}

*Letras iguais, na mesma coluna não apresentaram diferença significativa conforme determinado pelo teste do qui-quadrado ( $\mathrm{p}>0,05$ ). Fonte: Autores (2021).

Outras situações durante a pandemia, exigiram dos gestores das UAN medidas rápidas para evitar surpresas e outros gastos desnecessários. Um deles foi quanto ao desperdício de matéria prima, causado pela paralisação das atividades em virtude das medidas de contenção da Covid-19, que foi verificada em 39\% dos estabelecimentos pesquisados. Entretanto, a perda de matéria prima foi relatada em um maior número $(\mathrm{p}<0,05)$ de estabelecimentos comerciais que $\mathrm{o}$ setor corporativo/industrial. Essa diferença, pode ser explicada pela dificuldade do controle do estoque pelos gestores do segmento comercial, pois os decretos de paralisação das atividades eram impostos de forma repentina, podendo ainda serem postergados. 
O cancelamento da entrega de matérias primas pelos fornecedores, relatada por $21,9 \%$ das UAN pode ser explicada pela alteração na cadeia de alimentos no mundo todo. Muitas indústrias de alimentos tiveram suas linhas de produção paralisadas ou reduzidas, o que gerou pouca produção e atrasos nas entregas. Desse modo, com a matéria prima escassa e mais cara, os gestores dos estabelecimentos tiveram que contatar outros fornecedores. Logo observa que 23,8\% das UAN investigadas, relataram a época terem mudado de fornecedores durante a pandemia. Além disso, a mudança de fornecedores relatadas por um maior número de estabelecimentos comerciais em comparação ao corporativo/industrial ( $\mathrm{p}>0,05)$, pode ter ocorrido devido as paralizações constantes, obrigando-os a comprar alimentos sem agenda previa e de locais que tivessem a matéria prima a pronta entrega.

Ainda, com relação a mudanças de produção e na aquisição de matérias primas, observou-se aumento de gastos com materiais descartáveis relatada por $92,4 \%$ dos gestores. Esse acréscimo no consumo de objetos descartáveis, pode ter ocasionada pela inserção da prática de porcionar individualmente os alimentos e/ou utensílios para evitar a contaminação por gotículas de saliva durante a entrega de alimentos aos comensais no buffet, como também pelo aumento da atividade delivery.

Paralelamente às adaptações no quadro de funcionários, na produção e na aquisição de matérias primas as implementações das medidas higiênicos-sanitárias também tiveram que ser feitas de forma rápida. Essas modificações, como já comentado anteriormente, foi necessário para garantir a inocuidade dos alimentos e a proteção dos funcionários e clientes contra a Covid-19. Logo, os gestores passaram a seguir normas, determinadas por diferentes instituições, que os instruíam, principalmente ao uso de máscara para todos os colaboradores, afastamento de funcionários com sintomas gripais ou de Covid19, aumento da frequência da higienização de mãos e de superfícies de contato, e a promoção do distanciamento social entre as mesas na área de consumo dos alimentos (Nota técnica 47, 2020; Nota técnica 48, 2020; Nota técnica 49, 2020; Maciel et al., 2020). Desse modo, verificou-se que apenas 63,8\% dos estabelecimentos relataram efetuar as principais medidas higiênicossanitárias para conter a transmissão de Sars-CoV-2 (Tabela 6).

Tabela 6. Adesão das principais recomendações higiênicos sanitárias para a contenção de Covid-19 relatadas pelas UAN, do início da pandemia até 01 de setembro de 2021.

\begin{tabular}{|c|c|c|c|c|c|c|}
\hline Segmento & $\begin{array}{c}\text { Uso de } \\
\text { máscara por } \\
\text { todos os } \\
\text { colaboradores } \\
\%(n)\end{array}$ & $\begin{array}{c}\text { Intensificação } \\
\text { da higienização } \\
\text { de mãos e } \\
\text { superfícies } \\
\%(n)\end{array}$ & $\begin{array}{c}\text { Afastamento de } \\
\text { colaboradores } \\
\text { com sintomas } \\
\text { gripais ou de } \\
\text { Covid-19 } \\
\%(n)\end{array}$ & $\begin{array}{c}\text { Alteração na } \\
\text { organização do } \\
\text { estabeleciment } \\
\text { o para garantir } \\
\text { o } \\
\text { distanciamento } \\
\text { social dos } \\
\text { clientes } \\
\%(n)\end{array}$ & $\begin{array}{c}\text { Mudança no } \\
\text { sistema de } \\
\text { distribuição do } \\
\text { buffet, em } \\
\text { relação às } \\
\text { preparações que } \\
\text { ficavam expostas } \\
\%(n)\end{array}$ & $\begin{array}{c}\text { Todas } \\
\%(n)\end{array}$ \\
\hline $\begin{array}{c}\text { Corporativo/ } \\
\text { Industrial } \\
(73)\end{array}$ & $100(73)^{\mathrm{a}}$ & $98,6(72)^{a}$ & $82,2(60)^{\mathrm{a}}$ & $94,5(69)^{\mathrm{a}}$ & $86,3(63)^{\mathrm{a}}$ & $71,2(52)^{\mathrm{a}}$ \\
\hline $\begin{array}{c}\text { Comercial } \\
\text { (32) }\end{array}$ & $100(32)^{a}$ & $96,9(31)^{\mathrm{a}}$ & $65,6(21)^{\mathrm{a}}$ & $62,5(20)^{\mathrm{b}}$ & $59,4(19)^{\mathrm{b}}$ & $46,9(15)^{b}$ \\
\hline Total (105) & $100(105)$ & $98,1(103)$ & $77,1(81)$ & $84,8(89)$ & $78,1(82)$ & $63,8(67)$ \\
\hline
\end{tabular}

*Letras iguais, na mesma coluna não apresentaram diferença significativa conforme determinado pelo teste do qui-quadrado ( $\mathrm{p}>0,05$ ). Fonte: Autores (2021).

Entretanto, observa-se, conforme a Tabela 6, que mais UAN corporativas/industriais modificaram seus protocolos de higiene para conter a Covid-19, em comparação aos comerciais ( $p>0.05$ ), principalmente com relação a alteração na organização do estabelecimento para garantir o distanciamento social de clientes e quanto a mudanças no sistema de distribuição do buffet, em relação às preparações que ficavam expostas. Essa divergência, pode ser explicada pela pressão das 
empresas contratantes nos estabelecimentos corporativos/industriais, uma vez que essas também estavam sendo fiscalizadas pelo governo (Decreto 10.282, 2020). Logo, considerando que as UAN fazem parte das corporações, caso apresentassem inadequações, essas instituições e seus funcionários também seriam afetados. Em oposição, as UAN comerciais apresentavam baixo fluxo de clientes, e por isso realizaram as modificações aos poucos, conforme foram retornando normalmente as atividades. Em adição, os restaurantes comerciais parecem apresentaram menor porcentagem de adequações de boas práticas de fabricação em relação aos estabelecimentos institucionais e hotéis, uma vez que possuem menos nutricionistas e/ou responsáveis técnicos (Akutsu, Botelho, Camargo, Sávio \& Araújo, 2005).

Por fim, quanto as principais recomendações higiênicas sanitárias para a contenção de Covid-19, destaca-se positivamente que $100 \%$ das UAN investigadas relataram utilizar máscaras e $98,1 \%$ intensificaram a higienização de mãos e superfícies, diminuindo desse modo as principais vias de transmissão do SARS-CoV-2.

\section{Considerações Finais}

Restaurantes maiores, e com maior retorno de caixa parecem sobreviverem melhor ao retorno de ações após a volta de suas atividades. Entretanto, estabelecimentos menores e que não possuem marcas fortes no mercado e nem acesso a injeção de capital, sofrem para se reerguer financeiramente após a flexibilização das medidas de contenção da Covid-19 (Dube, Nhamo \& Chikodzi, 2021). Logo, alternativas que podem contribuir de forma positiva para estabelecimentos de pequeno e médio porte, após o afrouxamento das restrições da pandemia, é verificar as oportunidades de fusão e de downsizing, aderir a serviços de entrega, inovar o menu online e torná-lo atrativo, considerar vitais ao negócio o fluxo dos clientes de delivery (Brewer \& Sebby, 2021; Kim, Kim \& Wang, 2021), como também trazer acionistas da comunidade local (Dube et al., 2020).

Em adição, mais de 50\% das pessoas, possuem medo e/ou insegurança de contrair Sars-CoV-2, por meio de alimentos e/ou embalagens provenientes de restaurantes, ainda que esses não sejam os principais veículos de transmissão do novo vírus (Byrd et al., 2021). Desse modo, é necessário que os restaurantes se certifiquem a programas de boas práticas de fabricação, e mitiguem as incertezas dos clientes quanto a qualidade do preparo dos alimentos por meio de informações vinculadas de modo online (redes sociais e sites) e por folhetos e banners (Franco, Landgraf \& Pinto, 2020; Kim et al., 2021; Vig \& Agarwal, 2021).

Por fim, valorizar o trabalho dos colaboradores é de extrema importância, uma vez que esses viveram e vivem a incerteza do desemprego e de serem contaminados pela Covid-19, levando-os a exaustão emocional (Bufquin, Park, Back, Meira \& Hight, 2021; Chen \& Eyoun, 2021; Park, Hight, Bufquin, Meira \& Back, 2021). Desse modo, funcionários desestabilizados emocionalmente e insatisfeitos com o trabalho não conseguem ser produtivos (Bellet, De Neve \& Ward, 2019), prejudicando ainda mais o negócio.

Diante disso, configura-se que os fatores que geraram tantas falências de restaurantes não foi somente a pandemia, mas também as características inerentes a cada setor e a crises internas já implantadas (Madeira, Palrão \& Mendes, 2021) devido à falta de conhecimentos e práticas gerenciais competentes anteriores. Logo é necessário que os restaurantes fiquem atentos a mudanças de negócios e do padrão de compra dos consumidores ocasionadas pela pandemia, para preservar a saúde dos empreendimentos evitando levá-los à falência.

\section{Referências}

Akutsu, R. D. C., Botelho, R. A., Camargo, E. B., Sávio, K. E. O., \& Araújo, W. C. (2005). Adequação das boas práticas de fabricação em serviços de alimentação. Revista de Nutrição, 18, 419-427. https://doi.org/10.1590/S1415-52732005000300013

Associação Nacional de Restaurantes (2020). Pesquisa ANR aponta que mais de $60 \%$ dos bares e restaurantes já fizeram demissões por causa da crise do coronavírus. http://anrbrasil.org.br/pesquisa-anr-aponta-que-mais-de-60-dos-bares-e-restaurantes-ja-fizeram-demissoes-por-causa-da-crise-do-coronavirus/ 
Research, Society and Development, v. 10, n. 13, e40101320758, 2021

(CC BY 4.0) | ISSN 2525-3409 | DOI: http://dx.doi.org/10.33448/rsd-v10i13.20758

Associação Nacional de Restaurantes. Galunion. Instituto Foodservice Brasil (2020). Alimentação na pandemia - A visão dos operadores de foodservice https://galunion.com.br/links-galunion/materiais/pesquisa_alimentacao_na_pandemia_galunion_anr_operadores3.pdf?utm_campaign=download_3_pes qu isa _operadores\&utm_medium=email\&utm_source=RD+Station

Associação Nacional de Restaurantes. Galunion. Instituto Foodservice Brasil (2021). Alimentação na pandemia - A visão dos operadores de foodservice. https://galunion.com.br/links-galunion/materiais_galunion/20210512_Pesquisa_Operadores_ANR_Galunion_IFB_Sumario\%20(2).pdf?utm_campaign =resposta_automatica_da_landing_page_4_pesquisa_operadores_anrifbgalunion_-_sumario\&utm_medium=email\&utm_source=RD+Station

Aquino, E. M., Silveira, I. H., Pescarini, J. M., Aquino, R., Souza-Filho, J. A. D., Rocha, A. D. S., \& Lima, R. T. D. R. S. (2020). Medidas de distanciamento social no controle da pandemia de COVID-19: potenciais impactos e desafios no Brasil. Ciência \& Saúde Coletiva, 25, 2423-2446. https://doi.org/10.1590/1413-81232020256.1.10502020

Bellet, C., De Neve, J. E., \& Ward, G. (2019). Does employee happiness have an impact on productivity?. Saïd Business School WP, 13. https://dx.doi.org/10.2139/ssrn.3470734

Brewer, P., \& Sebby, A. G. (2021). The effect of online restaurant menus on consumers' purchase intentions during the COVID-19 pandemic. International Journal of Hospitality Management, 94, 102777. https://doi.org/10.1016/j.ijhm.2020.102777

Bucak, T., \& Yiğit, S. (2021). The future of the chef occupation and the food and beverage sector after the COVID-19 outbreak: Opinions of Turkish chefs. International Journal of Hospitality Management, 92, 102682. 10.1016/j.ijhm.2020.102682

Bufquin, D., Park, J. Y., Back, R. M., Meira, J. V. S., \& Hight, S. K. (2021). Employee work status, mental health, substance use, and career turnover intentions: An examination of restaurant employees during COVID-19. International Journal of Hospitality Management,93, 102764. https://doi.org/10.1016/j.ijhm.2020.102764

Byrd, K., Her, E., Fan, A., Almanza, B., Liu, Y., \& Leitch, S. (2021). Restaurants and COVID-19: What are consumers' risk perceptions about restaurant food and its packaging during the pandemic?. International Journal of Hospitality Management, 94, 102821. https://doi.org/10.1016/j.ijhm.2020.102821

Colares, L. G. T., \& Freitas, C. M. D. (2007). Processo de trabalho e saúde de trabalhadores de uma unidade de alimentação e nutrição: entre a prescrição e o real do trabalho. Cadernos de Saúde Pública, 23, 3011-3020. https://doi.org/10.1590/S0102-311X2007001200022

Chen, H., \& Eyoun, K. (2021). Do mindfulness and perceived organizational support work? Fear of COVID-19 on restaurant frontline employees' job insecurity and emotional exhaustion. International Journal of Hospitality Management, 94, 102850. https://doi.org/10.1016/j.ijhm.2020.102850

Cunha, D. T. D., Rosso, V. V. D., \& Stedefeldt, E. (2018). Food safety performance and risk of food services from different natures and the role of nutritionist as food safety leader. Ciencia \& saude coletiva, 23, 4033-4042. https://doi.org/10.1590/1413-812320182312.21042016

Da Silva, H. L. M., Carvalho, D. V., \& Mendes, A. E. P. (2021). Medidas preventivas para o enfrentamento da covid-19: relato de experiência em um serviço de alimentação institucional na cidade de Fortaleza-CE. Conexões-Ciência e Tecnologia, 15, 021010. https://doi.org/10.21439/conexoes.v15i0.1987

Decreto $\mathrm{n}^{\circ}$ 10.282, de 20 de março de 2020 (2020). Regulamenta a lei ${ }^{\circ} 13.979$, de 6 de fevereiro de 2020, para definir os serviços públicos e as atividades essenciais. Presidência da República. http://www.planalto.gov.br/ccivil_03/_ato2019-2022/2020/decreto/D10282.htm

Dube, K., Nhamo, G., \& Chikodzi, D. (2021). COVID-19 cripples global restaurant and hospitality industry. Current Issues in Tourism, 24(11), 1487-1490. https://doi.org/10.1080/13683500.2020.1773416

Franco, B. D. G. D. M., Landgraf, M., \& Pinto, U. M. (2020). Alimentos, Sars-CoV-2 e Covid-19: contato possível, transmissão improvável. Estudos Avançados, 34, 189-202. https://doi.org/10.1590/s0103-4014.2020.34100.012

Hakim, M. P., Zanetta, L. D. A., \& da Cunha, D. T. (2021). Should I stay, or should I go? Consumers' perceived risk and intention to visit restaurants during the COVID-19 pandemic in Brazil. Food Research International, 141, 110152. https://doi.org/10.1016/j.foodres.2021.110152

Kampf, G., Todt, D., Pfaender, S., \& Steinmann, E. (2020). Persistence of coronaviruses on inanimate surfaces and their inactivation with biocidal agents. Journal of hospital infection, 104(3), 246-251. https://doi.org/10.1016/j.jhin.2020.01.022

Kim, J., Kim, J., \& Wang, Y. (2021). Uncertainty risks and strategic reaction of restaurant firms amid COVID-19: Evidence from China. International Journal of Hospitality Management, 92, 102752. https://doi.org/10.1016/j.ijhm.2020.102752

Kumar, M., Taki, K., Gahlot, R., Sharma, A., \& Dhangar, K. (2020). A chronicle of SARS-CoV-2: Part-I-Epidemiology, diagnosis, prognosis, transmission and treatment. Science of The Total Environment, 734, 139278. https://doi.org/10.1016/j.scitotenv.2020.139278

Lei $\mathrm{n}^{\circ}$ 8.234, 17 de setembro de 1991 (1991). Regulamenta a profissão de Nutricionista e determina outras providências. Presidência da República. http://www.planalto.gov.br/ccivil_03/leis/1989_1994/18234.htm\#: :text=LEI\%20No\%208.234\%2C\%20DE,eu\%20sanciono\%20a\%20seguinte\%20lei\%3A\&te $\mathrm{xt}=6.206 \% 2 \mathrm{C} \% 20 \mathrm{de} \% 207 \% 20 \mathrm{de} \% 20$ maio, $20 \% 20 \mathrm{de} \% 20$ outubro $\% 20 \mathrm{de} \% 201978$

Lei ${ }^{\circ} 13.979$, de 6 de fevereiro de 2020 (2020). Dispõe sobre as medidas para enfrentamento da emergência de saúde pública de importância internacional decorrente do coronavírus responsável pelo surto de 2019. Presidência da República. Recuperado de https://www.in.gov.br/en/web/dou/-/lei-n- 13.979-de-6de-fevereiro-de-2020-242078735

Maciel, A. P., Lavareda, C. M. V., Silva, E. B., Mota, E. R. S., Oliveira, E. V., Silva, E. C., \& Mendonça, X. M. F. D. (2020). Boas práticas em serviços de alimentação coletiva: estratégias para o enfrentamento da covid-19. Recuperado de https://livroaberto.ufpa.br/jspui/handle/prefix/942

Madeira, A., Palrão, T., \& Mendes, A. S. (2021). The Impact of Pandemic Crisis on the Restaurant Business. Sustainability, 13(1), 40. https://doi.org/10.3390/su13010040 
Research, Society and Development, v. 10, n. 13, e40101320758, 2021

(CC BY 4.0) | ISSN 2525-3409 | DOI: http://dx.doi.org/10.33448/rsd-v10i13.20758

Medida Provisória $\mathrm{n}^{\circ}$ 927, de 22 de março de 2020 (2020). Dispõe sobre as medidas trabalhistas para enfrentamento do estado de calamidade pública reconhecido pelo Decreto Legislativo no 6, de 20 de março de 2020, e da emergência de saúde pública de importância internacional decorrente do coronavírus (Covid-19), e dá outras providências. Presidência da República. https://www.in.gov.br/en/web/dou/-/medida-provisoria-n-927-de-22-de-marco-de-2020249098775

Medida provisória $\mathrm{n}^{\circ}$ 936, de $1^{\circ}$ de abril de 2020 (2020). Institui o Programa Emergencial de Manutenção do Emprego e da Renda e dispõe sobre medidas trabalhistas complementares para enfrentamento do estado de calamidade pública reconhecido pelo Decreto Legislativo $\mathrm{n}^{\circ} 6$, de 20 de março de 2020 , e da emergência de saúde pública de importância internacional decorrente do coronavírus (covid-19), de que trata a Lei nº 13.979, de 6 de fevereiro de 2020, e dá outras providências. Presidência da República. https://www.in.gov.br/en/web/dou/-/medida-provisoria-n-936-de-1-de-abril-de-2020-250711934

Moraes, R. F. D., Silva, L. L. S., \& Toscano, C. M. (2020). Covid-19 e medidas de distanciamento social no Brasil: análise comparativa dos planos estaduais de flexibilização. http://repositorio.ipea.gov.br/bitstream/11058/10165/1/NT_25_Dinte_Covid19MedDistSocPlaEstFlex.pdf

National Restaurant Association (2020). Industry research. https://restaurant.org/manage-my-restaurant/business-operations/covid19/research/industryresearch

Nota técnica $\mathrm{n}^{\circ} 49$, de 2 de junho de 2020 (2020). Orientações para os serviços de alimentação com atendimento direto ao cliente durante a pandemia de Covid-19. Agência Nacional de Vigilância Sanitária. http://www.vigilanciasanitaria.sc.gov.br/index.php/149-noticias/noticias-2020/1148-anvisa-esclarecesobre-o-novo-coronavirus-e-os-alimentos

Nota técnica $\mathrm{n}^{\circ}$ 47, de 3 de junho de 2020 (2020). Uso de luvas e máscaras em estabelecimentos da área de alimentos no contexto do enfrentamento ao COVID-19. Agência Nacional de Vigilância Sanitária. http://www.vigilanciasanitaria.sc.gov.br/index.php/149-noticias/noticias-2020/1148-anvisa-esclarecesobre-o-novo-coronavirus-e-os-alimentos

Nota técnica $\mathrm{n}^{\circ}$ 48, de 5 de junho de 2020 (2020). Documento orientativo para produção segura de alimentos durante a pandemia de Covid-19. Agência Nacional de Vigilância Sanitária. http://www.vigilanciasanitaria.sc.gov.br/index.php/149-noticias/noticias-2020/1148-anvisa-esclarece-sobre-o-novocoronavirus-e-os-alimentos

Oliveira, T. C., Abranches, M. V., \& Lana, R. M. (2020). (In) Segurança alimentar no contexto da pandemia por SARS-CoV-2. Cadernos de Saúde Pública, 36, e00055220. https://doi.org/10.1590/0102-311X00055220

Ozili, P. K., \& Arun, T. (2020). Spillover of COVID-19: impact on the Global Economy. Available at SSRN 3562570. https://dx.doi.org/10.2139/ssrn.3562570

Park, J. Y., Hight, S. K., Bufquin, D., Meira, J. V. S, \& Back, R. M. (2021). An examination of restaurant employees’ work-life outlook: The influence of support systems during COVID-19. International Journal of Hospitality Management, 97, 102992. https://doi.org/10.1016/j.ijhm.2021.102992

Resolução da Diretoria Colegiada n² 216, de 15 de setembro de 2004 (2004). Estabelece procedimentos de boas práticas para serviço de alimentação, garantindo as condições higiênico-sanitárias do alimento preparado. Diário Oficial da União, Brasília. https://bvsms.saude.gov.br/bvs/saudelegis/anvisa/2004/res0216_15_09_2004.htm

Sant'Ana, H. M. P. (2012). Planejamento físico-funcional de unidades de alimentação e nutrição. Editora Rubio.

Song, H. J., Yeon, J., \& Lee, S. (2021). Impact of the COVID-19 pandemic: Evidence from the US restaurant industry. International Journal of Hospitality Management, 92, 102702. 10.1016/ j.ijhm.2020.102702

Vig, S., \& Agarwal, R. N. (2021). Repercussions of COVID-19 on small restaurant entrepreneurs: The Indian context. Strategic Change, 30(2), 145-152. https://doi.org/10.1002/jsc.2398

VoPham, T., Weaver, M. D., Hart, J. E., Ton, M., White, E., \& Newcomb, P. A. (2020). Effect of social distancing on COVID-19 incidence and mortality in the US. 10.1101/2020.06.10.20127589 